\title{
Anabases
}

\section{Aux origines poétiques de la couleur : pour une approche littéraire des lexiques chromatiques anciens. L'exemple des élégiaques latins et de leurs modèles grecs}

Lydia Pelletier-Michaud

\section{OpenEdition}

\section{Journals}

Édition électronique

URL : http://journals.openedition.org/anabases/5673

DOI : $10.4000 /$ anabases.5673

ISSN : 2256-9421

\section{Éditeur}

E.R.A.S.M.E.

Édition imprimée

Date de publication : 2 mai 2016

Pagination : 278-289

ISSN : $1774-4296$

\section{Référence électronique}

Lydia Pelletier-Michaud, « Aux origines poétiques de la couleur : pour une approche littéraire des lexiques chromatiques anciens. L'exemple des élégiaques latins et de leurs modèles grecs », Anabases [En ligne], 23 | 2016, mis en ligne le 02 mai 2019, consulté le 21 octobre 2019. URL : http:// journals.openedition.org/anabases/5673 ; DOI : 10.4000/anabases.5673

Ce document a été généré automatiquement le 21 octobre 2019

(c) Anabases 


\title{
Aux origines poétiques de la couleur : pour une approche littéraire des lexiques chromatiques anciens. L'exemple des élégiaques latins et de leurs modèles grecs
}

\author{
Lydia Pelletier-Michaud
}

\section{Les couleurs et l'Antiquité : le grand renouveau}

1 Depuis une bonne décennie, plusieurs questions touchant le chromatisme dans le monde gréco-romain font l'objet d'approches multidisciplinaires particulièrement stimulantes et fructueuses ${ }^{18}$; on pense notamment aux études récentes portant sur la polychromie de la sculpture antique ${ }^{19}$, grâce auxquelles l'idée erronée d'un art grécoromain tout en blancheur a pu être définitivement rejetée pour révéler une esthétique riche en contrastes et en teintes saturées. Un intérêt particulier pour l'usage des termes de couleur dans les textes de médecine se fait par ailleurs sentir, notamment dans le corpus hippocratique, dont on souligne la grande diversité lexicale et la précision avec lesquelles ses auteurs ont formulé des indications chromatiques ${ }^{20}$.

\section{La dimension littéraire, un aspect négligé}

2 Pourtant, la question qui a d'abord enflammé les débats, celle du vocabulaire de la couleur chez les poètes anciens, demeure toujours aussi épineuse. Si les textes poétiques, certes, sont mis à profit avec succès pour reconstituer le " paysage sensible des Grecs anciens ${ }^{21}$ ", les termes de couleur demeurent somme toute peu étudiés en tant qu'éléments du discours. Les écrits théoriques sur la couleur, ou encore l'usage scientifique du lexique chromatique, attirent avant tout l'intérêt des chercheurs : on 
tente de comprendre comment les Anciens concevaient la couleur, comment ils interprétaient les phénomènes chromatiques et de quelle manière ils classifiaient les nuances ${ }^{22}$. Le problème de la couleur dans le langage aurait-il été résolu en écartant la question littéraire?

\section{La nécessité d'une nouvelle remise en question}

L'époque semble aujourd'hui lointaine où dominaient encore les théories évolutionnistes au nom desquelles on allait jusqu'à évoquer, pour expliquer les particularités du vocabulaire homérique, une différence physiologique entre Anciens et Modernes $^{23}$ : l'hypothèse $\mathrm{du}$ daltonisme des Grecs de l'époque d'Homère fait aujourd'hui sourire ${ }^{24}$. On peut affirmer que l'on s'intéresse désormais aux systèmes de pensée anciens avec beaucoup plus d'ouverture, admettant d'emblée qu'il n'est pas pertinent de les examiner à l'aune des catégories modernes. Ainsi a-t-on supposé que les Grecs de l'époque archaïque étaient peut-être plus sensibles aux contrastes pâle/ foncé et moins attentifs aux nuances, ou encore que leur lexique ne leur permettait pas de désigner la couleur isolée des autres sensations concomitantes ${ }^{25}$. Par ailleurs, on a admis que " percevoir les couleurs et les nommer sont deux choses distinctes ${ }^{26}$ ", que la langue poétique a ses propres codes et que l'on ne peut attendre du poète que la précision chromatique soit son premier souci.

4 Mais la différence culturelle et les particularités du langage poétique suffisent-elles à expliquer la difficulté de traduire les termes de couleur et leur aspect parfois si déroutant ? Ne risque-t-on pas, en le posant de cette manière, de simplement déplacer le problème? Avant de s'intéresser aux éventuelles particularités des langues anciennes et du langage poétique relatives aux termes de couleur, il apparait en effet nécessaire de s'interroger d'abord sur la nature particulière de ce type de vocabulaire.

\section{La vraie nature du lexique chromatique}

5 En examinant avec attention les discours portant sur la couleur dans le langage, on constate rapidement qu'une idée les domine : les termes et expressions de couleur auraient pour utilité première de décrire avec le plus d'acuité possible les sensations visuelles - réelles ou imaginées - de celui qui en fait usage, et ce d'abord en termes de nuance. Cette idée n'est pas toujours exprimée mais, perçue comme une évidence, elle est souvent admise de façon implicite ${ }^{27}$.

Or si l'on abordait la littérature moderne avec un semblable a priori, la même impression d'étrangeté ne manquerait pas de s'en dégager. Certes, les poètes français ont évoqué l'azur des flots ${ }^{28}$; mais Chateaubriand a aussi chanté « le vert changeant des mers étincelantes ${ }^{29}$ " et contemplé les "froncis grisâtres » de l'Adriatique ${ }^{30}$; Verlaine, ses « airs bleus, roses, gris et verts ${ }^{31}$ » et son eau qui le soir devient « plus noire $e^{32}$ "; Beauchemin a célébré la " mer fauve ${ }^{33}$ ", et Hugo, son " flot d'argent ${ }^{34}$ ». Cette variété ne choque personne, bien au contraire ; pourtant, on n'hésite pas à parler d'incohérence lorsque l'on découvre chez Homère une mer "semblable au vin "

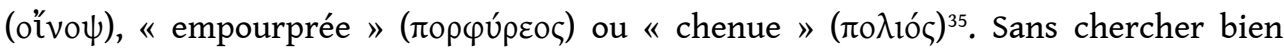
entendu à nier les différences évidentes entre la langue poétique homérique et celle des 
auteurs d'expression française des derniers siècles, ce parallèle permet de mettre en évidence les attentes non formulées des chercheurs face au texte ancien.

7 Les particularités de la langue des poètes ${ }^{36}$, certes, doivent être prises en compte ; nous y reviendrons. Toutefois, la licence poétique ne suffit pas à expliquer les écarts entre le texte homérique et ce que les chercheurs y ont cherché en vain - par exemple, une mer " bleue ${ }^{37}$ »-, écarts que l'on ne peut non plus attribuer à " l'influence des émotions " qui, selon J. Andrés ${ }^{38}$, auraient éloigné les termes de couleur de leur sens véritable.

8 Ce sens " premier ", qui serait synonyme d'objectivité, est en fait une illusion. En effet, si l'on prend le temps de s'interroger sur le vocabulaire de la couleur en français, tel qu'employé dans la langue parlée au quotidien, on s'aperçoit qu'il est dominé par des conventions et que sa fonction descriptive est somme toute limitée ${ }^{39}$. Pas davantage dans la prose que dans la langue parlée, les termes de couleur n'ont pour fonction première la désignation de nuances précises.

9 Cette inadéquation entre perception et expression n'affecte pas seulement la langue écrite ou parlée : à preuve, la couleur de l'eau n'est d'ailleurs pas davantage une évidence pour le peintre. Un journaliste du Guardian rapportait l'an dernier les paroles de l'artiste britannique D. Hockney à propos de l'exercice technique que représente sa série sur les piscines californiennes : " [...] they are an attempt to solve an almost insuperable formal problem : as he put it, water "can be any colour, it's movable, it has no set visual description", so how do you paint it $t^{40}$ ?"

10 Ni le peintre, ni le poète, pas plus que le prosateur ou le locuteur lambda ne se contentent de " reproduire » les couleurs qu'ils voient : tous s'adonnent, de façon consciente ou non, à un exercice d'interprétation et de représentation ${ }^{41}$. Nommer la couleur, la désigner par des mots, est en soi un processus empreint de subjectivité. Il faut donc abandonner la volonté de déterminer, à partir de matériel littéraire, et pour chaque terme de couleur grec ou latin, une nuance précise ${ }^{42}$.

\section{Les termes de couleur modernes : un produit de la culture... gréco-romaine}

11 Les remarques sur le vocabulaire de la couleur dans les textes anciens qui ont conduit à le caractériser comme différent, voire déficient ${ }^{43}$, s'appliquent donc aussi bien aux langues modernes ; réciproquement, les systèmes de classification que l'on considère d'emblée comme "modernes », c'est-à-dire post-newtoniens, sont en fait profondément enracinés dans la pensée ancienne.

Le modèle proposé par Newton, encore souvent perçu comme le triomphe de l'objectivité scientifique et de la raison sur le mystère de la couleur, implique une division des nuances du spectre en sept couleurs. Ce nombre, également retenu par Aristote pour décrire de façon générale la variété des couleurs, constitue un emprunt à la théorie musicale ${ }^{44}$. Conscients de ce rattachement à une tradition esthétique, certains chercheurs sont allés jusqu'à reprocher à l'homme de sciences britannique son manque de rationalisme $e^{45}$. Le continuum chromatique ne comporte pourtant pas de divisions naturelles déterminables par la déduction ou l'expérimentation : c'est dans la culture uniquement, et notamment à travers le langage, que ces catégories existent ${ }^{46}$. Newton, dans ce qu'il y aurait sans doute plutôt lieu de voir comme un acte d'humilité 
et d'honnêteté intellectuelle, a préféré le choix d'un nombre mystique à un modèle arbitraire - et il a proposé sept termes de couleur latins pour les nommer.

La présence, dans le langage, de dénominations plus générales qui servent entre autres à ordonner la variété des nuances selon certains groupes de couleurs prédominants, n'est pas non plus une " découverte " moderne ${ }^{47}:$ dans une discussion entre Fronton et Favorinus rapportée par Aulu-Gelle, les deux protagonistes énoncent clairement l'idée que certaines couleurs - en l'occurrence, le color rufus et le color uiridis - peuvent être désignées par divers termes dont certains en désignent des nuances particulières ${ }^{48}$. Toutefois, comme chez de nombreux chercheurs modernes, cette approche du lexique mène justement à l'interprétation fort douteuse d'un texte poétique. Après avoir écouté l'explication de Fronton, qui considère flauus comme un type de rouge (rufus) mêlé de vert (uiridis) et de blanc (albus), Favorinus déclare pouvoir enfin comprendre les beaux vers d'Ennius évoquant le " marbre blond " (flauus marmor) de la mer " céruléenne " (caeruleus) écumant sous les rames : selon cette explication, l'écume de la mer verdoyante (uirens) - Favorinus assimile le terme caeruleus à un type de uiridis - peut être qualifiée de flauus, puisque le terme désignerait un mélange de uiridis et d'albus ${ }^{49}$. L'idée des termes de couleur fondamentaux et les théories des mélanges sont peu utiles lorsqu'il s'agit de lire les poètes ; pourtant, comme en témoignent Fronton et Favorinus, ces derniers ne peuvent être exclus du débat sur le lexique des couleurs : tous les exemples qu'ils citent pour appuyer leur argumentation sont empruntés au corpus poétique. Il n'est pas anodin non plus que leur discussion prenne la forme d'une joute entre la langue grecque et la langue latine : précurseur des débats linguistiques modernes, ce texte du second siècle de notre ère montre que les difficultés théoriques liées à la couleur surgissent précisément lorsqu'il s'agit de chercher des équivalents d'une langue à l'autre.

Pour nourrir la réflexion sur la façon de comprendre le sens des termes de couleur chez les poètes, on relit avec profit la célèbre anecdote rapportée par Athénée et attribuée à Ion de $\mathrm{Chios}^{50}$. Dans cette histoire, un professeur de grammaire aux idées étroites est ridiculisé à cause de son manque de sensibilité littéraire : ayant entendu Sophocle citer les vers de Phrynichos pour louer les " joues de pourpre " d'un garçon séduisant, celui-ci s'indigne, jugeant la " teinture de pourpre " peu indiquée pour représenter le visage d'un jeune éphèbe. Sophocle réplique en citant plusieurs poètes et, sans en nommer l'auteur, mentionne l'" Aurore aux doigts de rose ", insinuant que pour son interlocuteur la célèbre expression homérique évoque sans doute des mains tachées de teinture. La distinction est clairement établie entre le sens que prennent les termes de couleur en poésie et celui que revêtent les mêmes mots dans un contexte plus pragmatique (matières, colorants, représentations picturales), avec à la clé un constat particulièrement important : pour comprendre ce vocabulaire, il faut connaître Homère. Lucien souligne lui aussi cette idée dans un dialogue où l'aède est désigné comme étant « le meilleur des peintres ", c'est-à-dire comme le maître des couleurs ${ }^{51}$.

\section{La primauté du texte poétique}

15 L'idée voulant que la prose scientifique ait davantage de valeur que la parole poétique pour la compréhension du lexique de la couleur doit donc impérativement être elle aussi remise en question. Elle pose un réel problème pour l'étude de ce vocabulaire dans les langues anciennes : d'abord, parce que les témoignages auxquels nous avons 
accès sont précisément des textes littéraires - et pour l'époque d'Homère, les vers d'un seul poète ; mais il faut également tenir compte du fait que, pour les Anciens, la poésie est parole de vérité. Ainsi, rien ne leur est plus naturel que d'évoquer les vers d'un poète pour appuyer une théorie scientifique ${ }^{52}$ : l'auteur des Problèmes du corpus aristotélicien cite Homère pour prouver que l'airain est un élément froid ${ }^{53}$; chez Plutarque, le médecin Érasistrate prend Sappho comme référence pour prononcer le diagnostic d'un homme souffrant d'une passion maladive ${ }^{54}$; Sénèque choisit les vers d'Ovide pour illustrer l'infinie variété des couleurs de l'arc-en-ciel ${ }^{55}$.

\section{L'analyse intertextuelle pour cadre théorique}

Face à un objet d'étude offrant aussi peu de prise dans le réel, reste un défi de taille à surmonter : celui du cadre théorique. En effet, si l'on aborde les textes sans autre conviction que celle de la subjectivité des termes de couleur, le danger est grand de glisser vers la rhétorique des symboles, autorisant le lecteur à chercher dans les intentions de l'auteur le reflet de ses propres réseaux d'association.

Pourtant, le caractère subjectif du vocabulaire chromatique ne doit pas être perçu comme un obstacle à son étude en contexte poétique : au contraire, la seule façon de l'aborder de manière sérieuse est de prendre en compte cette nature particulière. Autant il importe d'envisager l'étude des textes en considérant leur cadre historique, autant il s'avère primordial d'embrasser leur nature poétique pour pouvoir en faire des objets de l'histoire. L'analyse littéraire, c'est-à-dire la recherche du sens des termes dans toute la complexité de leur contexte d'utilisation, doit donc demeurer notre premier outil ; dans le cadre de notre thèse de doctorat ${ }^{56}$, cette analyse a été menée en concentrant notre attention sur un aspect particulier : les liens de parenté entre les textes.

\section{L'histoire des couleurs à travers les figures littéraires : du grec au latin}

Les termes de couleur, ces mots que l'on utilise pour tenter de décrire une réalité intangible, mais bien perceptible, sont définis en grande partie par leur histoire poétique. Ils ont acquis leur sens au fil des divers rôles que leur ont fait jouer les auteurs qui en ont usé par le passé. Il apparaît ainsi que le vocabulaire latin de la couleur ne peut se comprendre pleinement qu'à travers une étude des textes prenant en compte leurs modèles grecs. En effet, la littérature latine prend racine dans un terreau hellénique : elle se définit en prenant appui sur un fonds littéraire dominé par Homère et ancre le sens de ses tropes dans la référence au texte grec.

Cela est particulièrement vrai pour le corpus sur lequel nous avons concentré nos recherches, soit les textes des élégiaques romains (Ovide, Properce, Tibulle et le corpus Tibullianum) et ceux de Catulle, qui fut l'un de leurs principaux modèles et qui joue souvent un rôle d'intermédiaire entre les auteurs grecs et les augustéens. En effet, à l'instar des poètes alexandrins, les poetae noui et les élégiaques pratiquent le jeu érudit de la réécriture. S'adressant à un public qui connaît le grec et, surtout, les poètes grecs, ces auteurs mobilisent constamment ce fonds culturel commun. Il ne s'agit pas pour eux de présenter une traduction du texte préexistant, mais de prendre appui sur des 
vers bien connus pour leur donner une forme et un sens nouveaux et originaux. Pour bien comprendre la signification que les poètes latins donnent au vocabulaire complexe de la couleur, il était donc nécessaire d'appuyer notre analyse sur une lecture précise de leurs prédécesseurs grecs. Nous avons considéré avant tout les poètes alexandrins Théocrite et Callimaque, qui ont excercé une influence directe sur l'écriture des élégiaques, mais également, à travers eux, les poètes lyriques de l'époque archaïque et l'œuvre homérique, source d'un nombre étonnant de reprises comportant une allusion à la couleur.

Une fois mis en évidence les liens de parenté qui relient les œuvres entre elles, on voit soudain s'éclairer un complexe réseau de citations et on peut observer comment, au fil des réécritures, les images colorées successivement empruntées se transforment et évoluent.

\section{Rétablir le dialogue entre les poètes}

21 Une telle approche permet notamment de prendre en compte un aspect important et trop souvent négligé : la voix des auteurs. En effet, on ne peut envisager la littérature grecque ou latine comme un amas uniforme de termes et d'occurrences ${ }^{57}$. Trop souvent encore, nous nous sentons autorisés à rapprocher entre eux les fragments de divers auteurs, parfois issus d'époques et de contextes très éloignés, comme s'il s'agissait d'un tout cohérent correspondant à " la pensée grecque » ou "la pensée ancienne ", et oubliant qu'il s'agit de voix individuelles dont rien n'indique qu'elles concordaient.

Si par exemple on observe que le même terme de couleur est utilisé chez Homère et chez Callimaque dans un contexte semblable, on ne doit certes pas considérer ces deux occurrences comme d'égale valeur et de même signification. En effet, de nombreuses figures faisant appel au chromatisme que l'on peut qualifier de topoi chez les poètes alexandrins - et auxquels les auteurs latins rattachent leurs propres créations - se rapportent en fait à des passages homériques précis. On s'aperçoit alors que les textes plus tardifs donnent au mot ou à l'expression un sens qui dépend directement de l'utilisation qu'en fait Homère. Parler de continuité dans l'utilisation poétique du terme en question, sans prendre en compte la généalogie des réécritures, s'avère alors aberrant. C'est le cas, par exemple, de l'adjectif кuóveoc, dont on considère habituellement qu'il est, dès les textes d'Homère, la " couleur du deuil ${ }^{58}$ ». Pourtant, en rapprochant les diverses occurrences du terme dans l'Iliade et l'odyssée, on s'aperçoit que l'adjectif et ses dérivés, qui désignent sans doute un bleu sombre, sont bien davantage associés à la puissance divine. Or un passage de l'Iliade a davantage marqué les esprits : les poètes grecs et latins, dès la période archaïque et jusqu'à l'époque augustéenne, y ont fait de nombreuses allusions. Il s'agit des vers dans lesquels Thétis, sachant qu'elle ne pourra plus retarder la mort de son fils, revêt un voile ainsi qualifié pour se rendre chez Zeus ${ }^{59}$; c'est en référence à ce passage bien précis, où le voile de la puissante déesse, désarmée face à Zeus, apparaît comme un voile de deuil tout noir

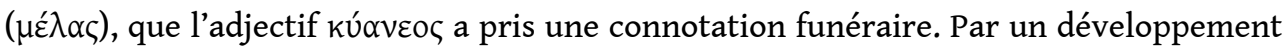
parallèle, Thétis est devenue une déesse "céruléenne " (caerulea) pour les poètes latins. Il paraît d'ailleurs fort plausible que son élément, la mer, ait été associé avec elle à des adjectifs chromatiques qui, en français, ont donné naissance au topos - car c'en est un - de la « mer bleue ». Ainsi, cette fameuse absente des épopées archaïques pourrait fort bien trouver son origine chez Homère. 
peut ainsi identifier, concentrées dans certaines expressions fixées par les poètes latins, des références succinctes à de nombreux passages homériques qui n'ont, eux, rien de formulaire, mais correspondent plutôt à un usage riche, polysémique, parfois même oxymorique, des termes de couleur.

\section{Un travail d'adaptation et de réappropriation}

Il ne s'agit donc pas, pour les poètes latins, de simplement " traduire " les termes grecs. En atteste l'exemple du terme pallidus et de l'utilisation spécifique qu'en font les élégiaques latins, et en particulier Ovide. Cet adjectif est utilisé par de nombreux auteurs latins pour rendre le sens du grec $\omega$ xpó et de termes apparentés. En parlant du teint d'un homme, symptôme d'une perte de contenance face à une menace extérieure. L'homme qui devient $\omega$ xpó ne sait pas faire face au danger : cette couleur révèle le lâche, figure opposée à celle du bon guerrier. Chez les élégiaques, pallidus devient au contraire une marque de valeur : le pallor de l'amant atteste la sincérité de ses sentiments ${ }^{60}$. On pourrait croire qu'il s'agit simplement d'autodérision ; mais en y regardant de plus près, on comprend que le " pallidus " d'Ovide n'est pas celui de Suétone. Il ne traduit pas la pâleur $\omega$ xpó du lâche, mais celle de l'homme véritablement confronté à la mort ou à la puissance divine : une saine pâleur, en quelque sorte, parente de la crainte

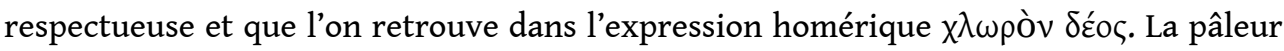
$\chi \lambda \omega \rho \alpha$ se rencontre aussi chez Sappho, dont le célèbre poème transmis par Longin

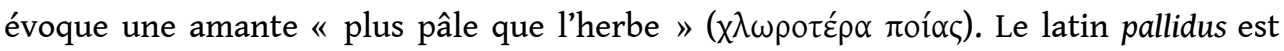
donc utilisé chez les élégiaques pour rendre l'aspect trouble de $\chi \lambda \omega \rho o ́ s \varsigma^{61}$, prenant une connotation méliorative inédite. Or cet adjectif grec désigne aussi l'aspect de la végétation croissante, associé alors à la vigueur, la vitalité et la fraîcheur ; en latin, c'est un autre adjectif, uiridis, qui reprend ce réseau d'associations affectives.

\section{La poésie comme outil de l'abstraction}

L'étude des reprises d'images colorées d'un texte à l'autre permet également d'observer le phénomène d'accumulation de comparants. Les poètes latins s'adonnent en effet à une sorte de jeu érudit consistant à rapprocher entre elles des expressions et des images issues de divers auteurs, un moyen de se réclamer de plusieurs modèles à la fois et de célébrer la richesse de leur héritage poétique. À titre d'exemple, on peut citer les enchaînements de comparaisons particulièrement développées auxquels les trois élégiaques ont recours pour illustrer le rougissement d'un visage, le plus souvent l'expression d'une pudeur noble. On peut retracer l'origine de ces comparaisons à un tout autre type de rougissement, celui du corps blessé de Ménélas décrit par Homère, alors que son sang se répand sur sa cuisse : l'aède compare alors la peau du guerrier à un ivoire teint d'écarlate, présent superbe destiné à un roi ${ }^{62}$. Chez Sappho, dans un contexte tout autre, une fleur pourpre et une pomme rougissante sont tour à tour évoquées en tant que comparants pour illustrer le sort d'une jeune fille dont la beauté intimide les prétendants éventuels ${ }^{63}$. Les deux modèles convergent à l'époque hellénistique pour donner naissance à deux types de portraits : celui du guerrier mourant et celui de la jeune épousée, deux destinées qui marquent l'apogée et le terme de la jeunesse caractérisée par le xpó $\alpha$ kó $\lambda o v$, le « beau teint ». Le rougissement est 
alors de plus en plus souvent évoqué comme le symptôme de bouleversements internes. Chez les élégiaques, toutes ces images fusionnent : on retrouve chez Ovide, côte à côte, l'ivoire teint et l'aurore d'Homère, les fleurs pourpres et les fruits rougissants de Sappho, les roses d'Anacréon et la fiancée catullienne, tous convoqués afin d'illustrer la perfection du teint de l'intraitable Corinne - un teint particulièrement séduisant puisqu'il évoque la pureté morale d'une innocente jeune fille ${ }^{64}$.

Les poètes sont des cueilleurs de fleurs, des tresseurs de couronnes : ils rassemblent des comparants puisés chez différents modèles pour créer des images nouvelles et composites et ce processus d'accumulation semble participer à l'établissement de catégories-couleurs. En effet, lorsqu'il évoque le purpureus pudor de Corinne en le comparant à l'Aurore aux doigts de rose et à l'ivoire teint d'écarlate ${ }^{65}$, lorsqu'il dépeint le rubor d'Hyménée en le comparant au safran de sa robe ${ }^{66}$, ou encore lorsqu'il hésite entre le sang et le minium pour qualifier la couleur de la cire de ses tablettes ${ }^{67}$, Ovide établit une identité entre différents comparants et différents qualificatifs : il met en évidence leur seul dénominateur commun, qui est leur aspect rouge. Ceci suggère que la poésie joue un rôle important dans le phénomène d'abstraction de la couleur.

\section{Conclusion}

L'étude des chemins qu'empruntent les références au chromatisme à travers la réécriture montre que les termes de couleur n'ont jamais de traduction unique et fixe : pas plus entre le grec et le latin qu'entre le latin et le français, il n'existe jamais vraiment de correspondance exacte. On a vu en effet qu'un terme latin, pallidus, peut porter le sens de deux adjectifs grecs différents, $\chi \lambda \omega \rho o ́ \varsigma$ et $\omega$ xpós ; réciproquement, $\chi \lambda \omega$ oóc est rendu tantôt par pallidus, tantôt par uiridis.

L'enquète révèle également l'importance du rôle d'Homère dans la création et le maniement d'un lexique chromatique chez les poètes grecs comme latins. Même chez les poètes élégiaques, qui rejettent vigoureusement la forme longue et les thèmes de l'épopée, les allusions aux vers homériques sont omniprésentes. Aborder le vocabulaire de la couleur dans les textes anciens avec la conviction qu'Homère en a fait un usage déficient équivaut donc à se priver du principal repère dont nous disposons.

La poésie doit donc être réhabilitée comme terrain d'étude valide pour l'étude des termes de couleur : il importe de reconnaître ses affinités particulières avec le registre chromatique et de chercher à comprendre le rôle qu'elle peut jouer dans les processus qui régissent le phénomène de la lexicalisation de la couleur. Les poètes de l'Antiquité en ont sans doute beaucoup à nous apprendre sur nos propres façons de dire la couleur, dont la complexité nous échappe trop souvent. Dans ce domaine, il est grand temps de reconsidérer l'importance de notre héritage culturel gréco-latin : après tout, nous sommes aussi les enfants d'Homère. 


\section{NOTES}

18. Signalons notamment M. CARASTRO (ÉD.), L’ANTIQUITÉ EN COULEURS, GRENOBLE, 2009 ; J. PIGEAUD (ÉD.), LA COULEUR, LES COULEURS. XI ENTRETIENS DE LA GARENNE-LEMOT, RENNES, 2007 ; A. ROUVERET, S. DUBEL ET V. NAAS (ÉD.), COULEURS ET MATIÈRES DANS L'ANTIQUITÉ. TEXTES, TECHNIQUES ET PRATIQUES, PARIS, 2006 ; L. CLELAND, K. STEARS ET G. DAVIES (ÉD.), COLOUR IN THE ANCIENT MEDITERRANEAN WORLD, OXFORD, 2004 ; S. BETA ET M.M. SASSI (ÉD.), I COLORI NEL MONDO ANTICO, ESPERIENZE LINGUISTICHE E QUADRI SIMBOLICI, FIESOLE, 2003, AINSI QUE L. VILLARD (ÉD.), COULEURS ET VISION DANS L'ANTIQUITÉ CLASSIQUE, MONT-SAINT-AIGNAN, 2002.

19. Encore tout récemment ont paru deux nouveaux recueils entièrement consacrés à la question : P. LIVERANI ET U. SANTAMARIA (ÉD.), DIVERSAMENTE BIANCO. LA POLICROMIA DELla SCULTURA RoMANA, ROME, 2014 AINSI QUE J.S. øSTERGAARD ET A.M. NIELSEN (ÉD.), TRANSFORMATIONS. ANCIENT SCULPTURE IN COLOUR, COPENHAGUE, 2014 (EN LIEN AVEC L'EXPOSITION PRÉSENTÉE À LA NY CARLSBERG GLYPTOTEK). IL FAUT ÉGALEMENT MENTIONNER L'OUVRAGE DE P. JOCKEY, LE MYTHE DE LA GRÈCE BLANCHE. HISTOIRE D'UN RÊVE OCCIDENTAL, PARIS, 2013.

20. Cf. dans les recueils indiqués ci-dessus (n. 18) les contributions d'E. BARRA (2009), D’I. воEнм, DE V. BOUDON ET DE L. VILLARD (2002).

21. Cf. A. GRAND-CLÉMENT, LA FABRIQUE DES COULEURS. HISTOIRE DU PAYSAGE SENSIBLE DES GRECS ANCIENS, PARIS, 2011. L'AUTEURE, QUI CO-DIRIGEAIT NOS TRAVAUX, A ÉGALEMENT SIGNÉ DE NOMBREUX ARTICLES CONSACRÉS À LA QUESTION DU CHROMATISME DANS LA GRÈCE ARCHAÏQUE. INSPIRÉE NOTAMMENT PAR LES DÉMARCHES DE M. PASTOUREAU ET DE M.F. FERRINI - MAIS ÉGALEMENT INFLUENCÉE PAR L'APPROCHE DE L'HISTORIEN A.CORBIN, AUTEUR AUQUEL LA FORMULE « PAYSAGE SENSIBLE » RENVOIE -, CETTE HISTORIENNE HELLÉNISTE APPRÉHENDE LA QUESTION D'UN POINT DE VUE ANTHROPOLOGIQUE, ACCORDANT UNE GRANDE ATTENTION AUX ASPECTS CULTURELS ET SOCIAUX DE LA COULEUR.

22. Cf. l'étude, par ailleurs fort pertinente, de M. BRADLEY (COLOUR AND MEANING IN ANCIENT ROME, CAMBRIDGE ET NEW-YORK, 2009), CENTRÉE SUR LA NOTION DE COLOUR ET SUR SES ACCEPTIONS DANS LES DISCOURS THÉORIQUES.

23. Quoique controversées, les théories des linguistes B. BERLIN ET P. KAY NE SEMBLENT POURTANT PAS PRÈS D'ÊTRE ABANDONNÉES, LORS MÊME QU'ELLES SONT OUVERTEMENT ÉVOLUTIONNISTES.

24. Si l'on attribue faussement cette idée à J.W. VON GOETHE (ZUR FARBENLEHRE, TÜBINGEN, 1810), QUI EN RÉALITÉ SOULIGNAIT LES PARTICULARITÉS DU VOCABULAIRE CHROMATIQUE EN GREC ANCIEN DANS LE BUT DE RELATIVISER LES IDÉES DE NEWTON, F. NIETZSCHE (MORGENRÖTE, CHEMNITZ, 1881) L'A EN REVANCHE EXPRESSÉMENT FORMULÉE, AFFIRMANT QUE LES GRECS [ANCIENS] ÉTAIENT AVEUGLES AU BLEU ET AU VERT (« [IHNEN WAR] DAS AUGE FÜR BLAU UND GRÜN BLIND »). L'OPHTALMOLOGUE BELGE A. DE KEERSMAECKER (LE SENS DES COULEURS CHEZ HOMÈRE, BRUXELLES, 1883) CROYAIT PLUS VOLONTIERS À UN PROBLÈME VISUEL PROPRE À LA PERSONNE D’HOMÈRE. DANS SA MONOGRAPHIE CONSACRÉE À LA PERCEPTION DES COULEURS CHEZ LES GRECS ANCIENS, W. SCHULTZ (DAS FARBENEMPFINDUNGSSYSTEM DER HELLENEN, LEIPZIG, 1904) CONCLUT QUE LES HELLÈNES SOUFFRAIENT D'UNE FORME DE DALTONISME QUI LES RENDAIT AVEUGLES AU JAUNE ET AU BLEU (BLAUGELBBLIND).

25. E. IRWIN, COLOUR TERMS IN GREEK POETRY, TORONTO, 1974, PRÉSENTE CES DEUX HYPOTHÈSES DANS SON ÉTUDE

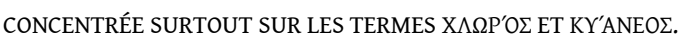

26. Cf. J. ANDRÉ, ÉTUDE SUR LES TERMES DE COULEUR DANS LA LANGUE LATINE, PARIS, 1949, P.19. LE FAIT D’AVOIR IMPOSÉ CETTE DISTINCTION CONSTITUE SANS DOUTE LE PLUS GRAND MÉRITE DE L'OUVRAGE DU LATINISTE.

27. M. PERRIN ( REGARDS CROISÉS SUR LA COULEUR, DE L'ANTIQUITÉ AU MOYEN ÂGE, AUTOUR DE QUELQUES NOTES DE LECTURE », IN BAGB, 2001.2, P. 156-157) FORMULE CETTE IDÉE QUE L'ON RETROUVE DÉJÀ CHEZ J. ANDRÉ (1949), P. 11.

28. A. DE MUSSET PARLE DU « FLOT D’AZUR » DES MERS CALMES (« À MON AMI ALFRED T. », IN PREMIÈRES POÉSIES, 1829) ; A. DE LAMARTINE ÉVOQUE SES « FLOTS D'OR ET D’AZUR » (ADIEUX À LA MER, 1840).

29. F.-R. DE CHATEAUBRIAND, « LA MER », IN TABLEAUX DE LA NATURE, VERS 1784-1790. 
30. F.-R. DE CHATEAUBRIAND, « SUR VENISE », IN MÉMOIRES D'OUTRE-TOMBE, 1809-1941.

31. P. VERLAINE, SAGESSE, EXTRAIT XV, 1889.

32. P. VERLAINE, « EN BATEAU », IN FÊTES GALANTES, 1869.

33. N. BEAUCHEMIN, « LA MER », IN LES FLORAISONS MATUTINALES, 1897.

34. V. HUGO, LES ORIENTALES, 1929.

35. Sur le vocabulaire de la mer en Grèce archaïque, cf. A. GRAND-CLÉMENT, « LA MER POURPRE : FAçONS GRECQUES DE VOIR EN COULEUR. REPRÉSENTATIONS LITTÉRAIRES DU CHROMATISME MARIN À LA PÉRIODE ARCHAÏQUE », IN PALLAS, 92 (2013), P. 143-161.

36. Nous entendons ici «langue poétique » au sens large, et non la forme versifiée, par opposition à la prose.

37. A. GRAND-CLÉMENT (2013), P.144, CRITIQUE AVEC RAISON LA DÉMARCHE D’A. CHRISTOL (« LES COULEURS DE LA MER », IN L. VILLARD ÉD. [2002], P. 29-44), QUI TRAHIT LA VOLONTÉ DE RETROUVER À TOUT PRIX UNE MER « BLEUE »

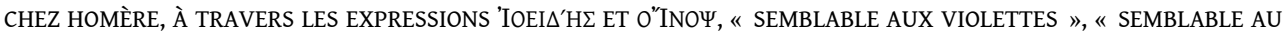
VIN ».

38. J. ANDRÉ (1949), P. 11, ÉVOQUAIT EN EFFET LE « PRISME DÉFORMANT » DES ÉMOTIONS.

39. Par exemple, les expressions du type « vin rouge ", " pin rouge » ou « peau rouge " se réfèrent à des systèmes de comparaison dont ne peut rendre compte un modèle au sein duquel le « rouge » correspondrait à une teinte précise. Par ailleurs, lorsqu'il s'agit de désigner la nuance d'un objet dont la couleur est indépendante de sa nature (e.g. " une écharpe violette »), il n'est pas rare que le consensus soit difficile à trouver : les termes de couleur n'ont pas de valeur absolue et leurs repères varient d'un individu à l'autre, même entre personnes parlant la même langue, issues d'une même culture et ayant un niveau d'éducation équivalent.

40. B. MORRISON, " David Hockney : the poets that make me paint ", in The Guardian, 24 janvier 2014.

41. Pour reprendre encore une fois les mots du peintre rapportés par le journaliste, « il n'existe pas de véritable copie » (" There is no such thing as a copy, really »).

42. Comme le rappelait L. WITTGENSTEIN (BEMERKUNGEN ÜBER DIE FARBEN, BERKELEY ET LOS ANGELES, 1977 [OUVRAGE POSTHUME], PARAGRAPHE 68), ON NE SAURAIT DÉFINIR QUELQUE COULEUR QUE CE SOIT AUTREMENT QU'EN PRÉSENTANT DES EXEMPLES CONCRETS.

43. Chez les philologues de la fin du $\mathrm{XIX}^{\mathrm{E}} \mathrm{S}$. ET DU XX $\mathrm{X}^{\mathrm{E}}$ S., TELS QUE W.E. GLADSTONE, M. PLATNAUER ET H. OSBORNE, LE VOCABULAIRE GREC DE LA COULEUR EST COURAMMENT APPELÉ « IMMATURE, PUÉRIL » (IMMATURE, JEJUNE), « LACUNAIRE » (DEFECTIVE) OU ENCORE « INCOHÉRENT » (INCONSISTENT).

44. Arstt. Sens., 442a12-25. La référence à Aristote n'est pas revendiquée par Newton, mais tous deux font appel à la même tradition esthétique.

45. M. Blay (Lumières sur les couleurs : le regard du physicien, Paris, 2001, p. 115-116) rapporte les critiques de C. Blanc (1867), choqué par le choix de Newton, jugé arbitraire, d'avoir inclus ce qui à son avis n'est qu'une nuance de bleu (l'indigo).

46. En effet, on peut diviser l'arc-en-ciel en autant de couleurs qu'on le veut : trois pour Aristote

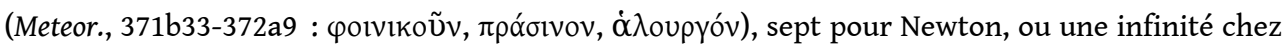
Sénèque (cf. ci-dessous, n. 55).

47. Cette idée est à la base de la théorie défendue par les linguistes B. BERLIN ET P. KAY (CF. CI-DESSUS, N. 23).

48. Gell. II, 26, 2-3. La discussion débute par un constat à propos de la difficulté de nommer la couleur : plura [...] sunt [...] in sensibus oculorum quam in uerbis uocibusque colorum discrimina, " la couleur revêt des aspects multiples ; les mots pour la nommer sont en revanche imprécis et peu nombreux ". Fronton énumère ensuite notamment au nombre des adjectifs exprimant la couleur rouge (rufus color) les termes fuluus, flauus, rubidus, poeniceus, rutilus, luteus et spadix.

49. Gell. II, 26, 12 et 21-23.

50. Ath. XIII, 81. 
51. Cf. Luc. Im. 8.

52. CEuvre poétique et œuvre scientifique ne sont d'ailleurs aucunement des catégories opposables dans l'Antiquité (on songe parmi de nombreux exemples à l'œuvre de Lucrèce).

53. Ps. Arstt. Probl. IX, 9, renvoie à Il. V, 75.

54. Plut. Dem. 38, renvoie à Sapph. 31 Voigt.

55. Sen. Nat. I, 3-15, renvoie à Ov. M. VI, 61-67.

56. « Évolution du sens des termes de couleur et de leur traitement poétique : l'élégie romaine et ses modèles grecs " (thèse préparée sous la direction d'A. Baudou et d'A. Grand-clément, soutenue à Québec le 16 novembre 2015).

57. Cf. l'approche de J. ANDRÉ (1949) ET, TOUT RÉCEMMENT, CELLE DE R.B. GOLDMAN (COLOR-TERMS IN SOCIAL AND CULTURAL CONTEXT IN ANCIENT ROME, PISCATAWAY, 2013). SELON CES AUTEURS, POÈTES ET PROSATEURS PEUVENT ÊTRE ENVISAGÉS D'UN MÊME REGARD; LA SECONDE NE JUGE PAS DAVANTAGE PERTINENT DE PRENDRE EN CONSIDÉRATION LA DIFFÉRENCE D’ÉPOQUE ENTRE LES TEXTES ABORDÉS (P. 7).

58. Cf. L. GERNET, « DÉNOMINATION ET PERCEPTION DES COULEURS CHEZ LES GRECS », IN PROBLÈMES DE LA COULEUR (I. MEYERSON ÉD.), PARIS, 1957, P. 313-327.

59. H. Il. XXIV, 93-94.

60. Cf. notamment Ov. A. A. I, 721-736.

61. À propos de cet adjectif et de sa signification complexe, cf. A. DIMAKOPOULOU, CHLÔRÊIS AÊDÔN, PÂLE ROSSIGNOL. UNE ÉTUDE SÉMANTIQUE, PARIS, 2010. DANS CE TEXTE ÉCRIT EN 1980 MAIS QUI N'A RIEN PERDU DE SON INTÉRÊT, L'AUTEURE EXPLORE AVEC SUCCÈS LES CONTOURS DE L'UNIVERS SÉMANTIQUE DE L'ADJECTIF X $\Lambda \Omega P^{\prime} O \Sigma$ CHEZ LES POÈTES DE LA GRÈCE ARCHAÏQUE.

62. H. Il. IV, 139-150.

63. Sapph. $105 \mathrm{a}$ et $105 \mathrm{~b}$ Voigt.

64. Cf. Ov. Am. II, 5, 33-42.

65. Cf. le passage mentionné dans la note précédente.

66. Ov. H. XXI, 163-170.

67. Ov. Am. I, 12, 11-12.

\section{AUTEUR}

\section{LYDIA PELLETIER-MICHAUD}

Docteur de l'Université Laval, Québec

lydia.pelletier.michaud@gmail.com 